\title{
Modern Optics and Photonics
}

Atoms and Structured Media 
This page intentionally left blank 
Modern Optics and Photonics Atoms and Structured Media

\author{
editors
}

Gagik Yu. Kryuchkyan

Yerevan State University, Armenia

\title{
Gagik G. Gurzadyan
}

Nanyang Technological University, Singapore

\section{Aram V. Papoyan}

Institute for Physical Research of the National Academy of Sciences, Armenia 
Published by

World Scientific Publishing Co. Pte. Ltd.

5 Toh Tuck Link, Singapore 596224

USA office: 27 Warren Street, Suite 401-402, Hackensack, NJ 07601

UK office: 57 Shelton Street, Covent Garden, London WC2H 9HE

\section{British Library Cataloguing-in-Publication Data}

A catalogue record for this book is available from the British Library.

\section{MODERN OPTICS AND PHOTONICS \\ Proceedings of the International Advanced Research Workshop}

Copyright $\odot 2010$ by World Scientific Publishing Co. Pte. Ltd.

All rights reserved. This book, or parts thereof, may not be reproduced in any form or by any means, electronic or mechanical, including photocopying, recording or any information storage and retrieval system now known or to be invented, without written permission from the Publisher.

For photocopying of material in this volume, please pay a copying fee through the Copyright Clearance Center, Inc., 222 Rosewood Drive, Danvers, MA 01923, USA. In this case permission to photocopy is not required from the publisher.

ISBN-13 978-981-4313-26-1

ISBN-10 981-4313-26-2

Printed in Singapore. 


\section{PREFACE}

The present volume includes plenary talks presented at the International Advanced Research Workshop on "Modern Problems in Optics and Photonics", MPOP-2009. The Workshop was held in Armenia, from 27 August to 2 September, 2009. Hosts were Yerevan State University and Institute for Physical Research of National Academy of Sciences. The Workshop was bestowed to the 90-th Anniversary of the Yerevan State University (http://www.ipr.sci.am/mpop/). It provided an opportunity to bring together experienced and young scientists on various facets of modern optics and photonics, to converse and consider the most recent developments in these areas.

Modern laser optics and nanoscience are perhaps the fastest growing areas of material research and technological development. Photonic science is concerned mainly with optical investigations elaborating the new fundamental concepts, the generation and technological utilization of photons. For the further development of optical and laser technologies the quest for new materials and principles for generation, transmission, modulation, signal processing, switching, amplification and manipulation of photons are necessary. When photonics merges with nanoscience and nanotechnology, spatial confinement considerably modifies light propagation and lightmatter interaction. It should also be noted that the impetuously growing directions of quantum information and quantum computing are primarily focused on the methods of quantum optics and novel composite materials. Therefore, it is expected that photonic technologies progressively gain importance in the 21st century.

Optics and laser research has a long lasting history in Armenia. In a rapidly changing field like modern optics and photonics it is not so easy exactly to formulate a standpoint of the field. Therefore, we as organizers of the workshop have tried to cover large area of topics and problems in this field. We hope that the workshop provides an impulse of the current activity at the research frontier of the areas.

The 22 papers are the end result of highly selective review process. They 
represent the state of the art in quantum optics and spectroscopy of the structured nonlinear media. This material is segregated into three chapters: Quantum optics, Structured materials and Photonic processes, Nonlinear optical effects and Spectroscopy.

Chapter I presents articles on quantum optical phenomena. In this chapter, the different aspects of light propagation in atomic and nuclear media, including phase control of light propagation through closed-loop atomic media and coherent control of nuclear forward scattering are discussed. In the other part, high-order harmonic generation with strong laser pulses employing electron ionization-recollision from highly charged ions is investigated in the relativistic regime. Generation of entangled light and superbunched photons via laser-multiparticle interactions as well as entanglement phenomena for matter waves is also studied. The Chapter contains discussion on irreversible photon transfer in an ensemble of -type atoms as well as a photonic diode. The results on applications of the frequency modulated (chirped) laser pulses in atomic physics are presented. This Chapter also elaborates on the investigation of quantum effects in a dissipative chaos where the counterpart of the semiclassical Poincaré section in quantum treatment is discussed. The prospect of utilizing quantum phases of light in a single atom driven by multiple different modes of photons is presented for possible quantum devices. Furthermore, the investigation of quantum adiabatic dynamics, where the external field moves slowly in time and is influenced by system's state (feedback) is presented. The Chapter also includes a meticulous analysis of several versions of Landau-Zener transition in nonlinear quantum systems.

Chapter II consists of papers mainly dedicated to structured nonlinear media, quantum dots and photonic devices. The multiple interactions in superlattice structures of nonlinear second-order material leading to the simultaneous quasi-phase matching of several parametric processes are discussed. A review and original studies of integrated photonic device structures with nanoscale features and sensitive applications are included in this chapter. One and two electronic and excitonic states in quantum dots with the non trivial geometries are discussed in the next article. The nanostructures are considered as the basics of novel photonic devices. In the other part, the properties of planar plasmonic structures and nonlinear metal-dielectric sub wavelength waveguides are investigated. The Chapter also involves material devoted to computer algebra study of structural and symmetry properties of discrete dynamical systems. A complete analysis and characterization of the bound and scattering states of two and three bosonic atoms in a one- 
dimensional optical lattice are presented. A vital phenomenon for atomic polaritons in a cavity array for the interaction of two-level atoms with quantized optical radiation in the strong coupling regime is also discussed.

Chapter III comprises articles on nonlinear optical effects and spectroscopy. The experimental investigations of formation of spectrally narrow optical resonances based on resonant absorption and fluorescence processes in an extremely thin vapor cells with smoothly variable thickness comparable with the resonant wavelength is presented. The nonlinear magnetooptical resonances in atomic rubidium in extremely thin cells are studied both experimentally and theoretically with high accuracy. The other contribution describes the modern problems of laser separation of isotopes in atomic vapors. The Chapter also incorporates both theoretical and experimental investigations of two-dimensional confined terahertz wave propagation in gap plasmon waveguide formed by two cylindrical surfaces. The complete characterization of the broadband similariton by means of the chirp measurement through the technique of spectral compression and frequency tuning in the sum-frequency generation process is presented in the last article.

We would like to express our sincere gratitude to the authors of the papers. We also thank the sponsors of the Workshop: State Committee of Science of Armenia, National Foundation of Science and Advanced Technologies (NFSAT), International Society of Optics Engineering (SPIE), Optical Society of Armenia (OSA). Sponsorship of EINST Technology, Pte Ltd (Singapore) and Analytical Technologies, Pte Ltd (Singapore) in publication of the present Proceedings is gratefully acknowledged. We would like to mention the valuable role of members of the Organizing and Program Committees, and, in particular, Diana Antonosyan for her generous contributions in organizing the conference and to the preparation of this volume.

Gagik Kryuchkyan

Yerevan State University and Institute for Physical Research, Armenia. kryuchkyan@ysu.am

Gagik Gurzadyan

Nanyang Technological University, Singapore.

gurzadyan@ntu.edu.sg

Aram Papoyan

Institute for Physical Research, Armenia.

papoyan@ipr.sci.am 
The International Workshop

"Modern Problems in Optics and Photonics-2009"

was organized by

Yerevan State University (Faculty of Physics)

National Academy of Sciences of Armenia

(Institute for Physical Research)

Russian-Armenian (Slavonic) State University

State Committee of Science of Armenia

Publication of the Proceedings is sponsored by

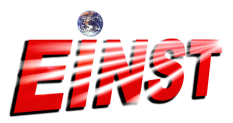

EINST Technology

Pte Ltd

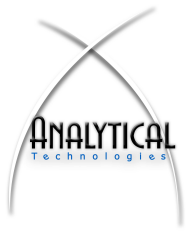

Analytical Technologies

Pte Ltd 

m.

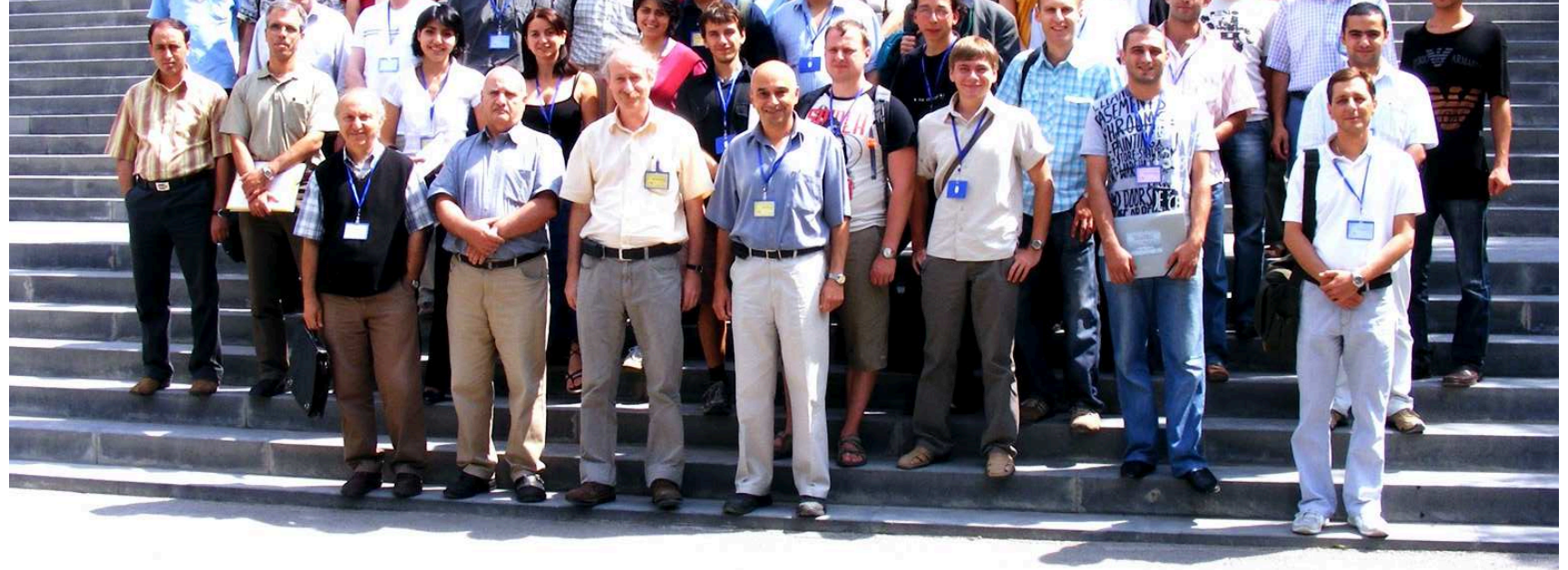




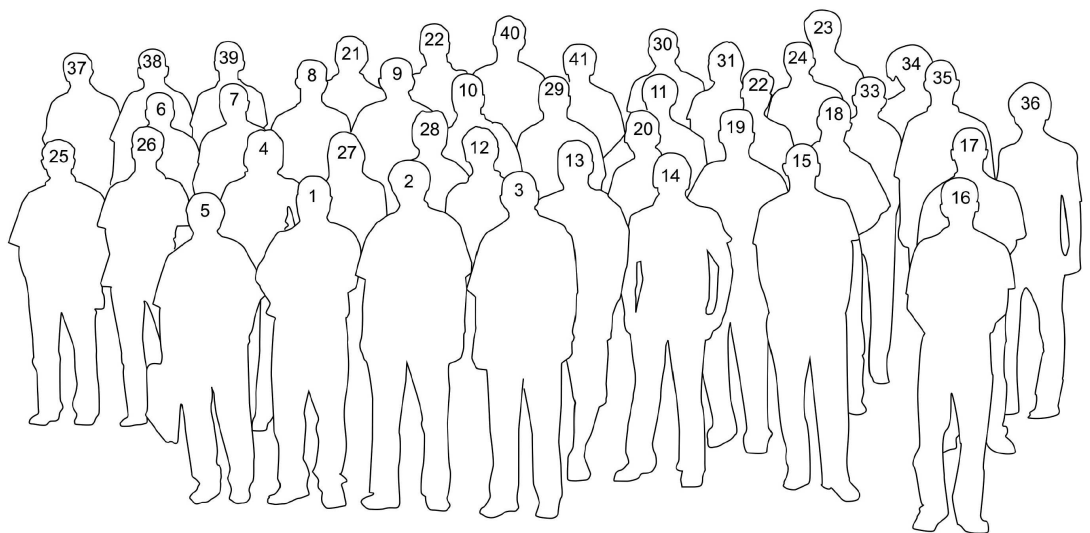

1. Gagik Kryuchkyan - Yerevan State University, Institute for Physical Research of NAS, Armenia. 2. Gagik Gurzadyan — Nanyang Technological University, Singapore; Technical University of Munich, Germany. 3. Aram Papoyan — Institute for Physical Research of NAS, Armenia. 4. Diana Antonosyan - Yerevan State University, Armenia. 5. David Sarkisyan - Institute for Physical Research of NAS, Armenia. 6. Karen Hatsagortsyan - Max-Planck Institute for Nuclear Physics, Germany. 7. Mihal Macovei - Max-Planck Institute for Nuclear Physics, Germany. 8. Jörg Evers Max-Planck Institute for Nuclear Physics, Germany. 9. Alexander Alodjants — Vladimir State University, Russia. 10. Aranya Bhattacharjee Max-Planck Für Physik Komplexer Systeme, Dresden, Germany; Department of Physics, University of Delhi, New Delhi, India. 11. Richard De La Rue - University of Glasgow, United Kingdom. 12. Yuriy Gladush - Institute of Spectroscopy, Ras, Russia. 13. Roman Noskov - Institute for Physics of Microstructures of RAS, Nizhny Novgorod, Russia. 14. Maxim Tsarev — Nizhny Novgorod State University, Russia. 15. Robert Sargayan - Institute for Physical Research of NAS, Armenia. 16. David Petrosyan - Institute of Electronic Structure \& Laser, Forth, Crete, Greece. 17. Gor Nikoghosyan - University of Kaiserslautern, Germany; Institute for Physical Research of NAS, Armenia. 18. Vahan Nanumyan - Yerevan State University, Armenia. 19. Armen Sargsyan — Institute for Physical Research of NAS, Armenia. 20. Simon Liebing - Institute für Theoretische Physic, Germany. 21. Misnik Kazaryan - P. N. Lebedev Physical Institute of the NAS, Russia. 22. Dariusz Łukaszewski - Warsaw University of Technology, Institute of Micromechanics and Photonics, Poland. 23. Linards Kalvans - Laser Centre of the University of Latvia, Riga, Latvia. 24. Raphael Drampyan - Institute for Physical Research of NAS, Armenia. 25. Behrooz Rezael - University of Tabriz, Research Institute for Applied Physics and Astronomy, Tabriz, Iran. 26. Aryan Hamid Reza — Research Institute for Applied Physics and Astronomy, Tabriz, Iran. 27. Narine Gevorgyan - Institute for Physical Research of NAS, Armenia. 28. Tatyana Buryakina — Institute of Biophotonics, National Yang-Ming University, Taiwan. 29. Emil Gazazyan - Institute for Physical Research of NAS, Armenia. 30. Razmik Unanyan - University of Kaiserslautern, Germany; Institute for Physical Research of NAS, Armenia. 31. Anna Shahinyan - Yerevan State University, Armenia. 32. Mushegh Rafayelyan - Yerevan State University, Armenia. 33. Vardan Gevorgyan - Institute for Physical Research of NAS, Armenia. 34. Anahil Gogyan - Institute for Physical Research of NAS, Armenia; Universite de Bourgogne, France. 35. Levon Yaghiazaryan — Yerevan State University, Armenia. 36. Andranik Hakobyan - Russian Amenian (Slavonic) University, Armenia. 37. Karapet Mkrtchyan — Yerevan Physics Institute, Armenia. 38. Vahagn Petrosyan - Joint Institute for Physical Researches, Russia. 39. Areg Meliksetyan — Yerevan State University, Armenia. 40. Karen Vardanyan - Institute for Physical Research of NAS, Armenia. 41. Hasmik Demirkhanyan — Institute for Physical Research of NAS, Armenia. 


\section{CONTENTS}

Preface $\quad$ v

PHOTO

$\begin{array}{lll}\text { Part A Quantum Optics } & 1\end{array}$

LIGHT PROPAGATION: FROM ATOMIC TO NUCLEAR QUANTUM OPTICS

J. Evers, R. Fleischhaker, A. Pálffy and C. H. Keitel

RELATIVISTIC HIGH-ORDER HARMONIC GENERATION

M. C. Kohler and K. Z. Hatsagortsyan

ENTANGLED LIGHT AND MATTER WAVES VIA NON-LINEAR INTERACTIONS

M. Macovei, G. Yu. Kryuchkyan and G.-X. Li

IRREVERSIBLE PHOTON TRANSFER IN AN ENSEMBLE OF $\Lambda$-TYPE ATOMS AND PHOTON DIODE

G. Nikoghosyan and M. Fleischhauer

DISSIPATIVE CHAOS IN QUANTUM DISTRIBUTIONS

T. V. Gevorgyan, S. B. Manvelyan, A. R. Shahinyan and G. Yu. Kryuchkyan

FREQUENCY CHIRPED LASER PULSES IN ATOMIC PHYSICS: COHERENT CONTROL OF INNER AND TRANSLATIONAL QUANTUM STATES

G. P. Djotyan, J. S. Bakos, Zs. Sörlei, G. Demeter, N. Sándor,

D. Dzsotjan, M. Á. Kedves, B. Ráczkevi, P. N. Ignácz and

J. Szigeti 
STRONGLY CORRELATED QUANTUM DYNAMICS OF MULTIMODE LIGHT COUPLED TO A TWOLEVEL ATOM IN A CAVITY

T. Kumar, A. B. Bhattacherjee and M. Mohan

FEEDBACK-DRIVEN ADIABATIC QUANTUM DYNAMICS 103 A. E. Allahverdyan and G. Mahler

LANDAU-ZENER TRANSITION IN NONLINEAR QUANTUM SYSTEMS

A. M. Ishkhanyan

Part B Photonic and Electronic Phenomena in Structured Media

MULTIPLE INTERACTIONS IN MULTILAYERED STRUCTURES OF NONLINEAR MATERIALS

D. A. Antonosyan and G. Yu. Kryuchkyan

INTEGRATED PHOTONIC DEVICE STRUCTURES WITH NANO-SCALE FEATURES: FOR SENSITIVE APPLICATIONS

R. M. De La Rue

ONE-, TWO-ELECTRONIC AND EXCITONIC STATES IN A QUANTUM DOTS WITH NONTRIVIAL GEOMETRIES: ADIABATIC DESCRIPTION

K. G. Dvoyan, E. M. Kazaryan and H. A. Sarkisyan

PLANAR PLASMONIC STRUCTURES AND NONLINEAR METAL-DIELECTRIC SUBWAVELENGTH WAVEGUIDES

A. R. Davoyan, I. V. Shadrivov and Yu. S. Kivshar

COMPUTER ALGEBRA STUDY OF STRUCTURAL AND SYMMETRY PROPERTIES OF DISCRETE DYNAMICAL SYSTEMS

V. V. Kornyak 
EXOTIC FEW-BODY BOUND STATES IN A LATTICE

$D$. Petrosyan and M. Valiente

SLOW LIGHT AND PHASE TRANSITION IN THE ARRAY OF ATOMIC POLARITONS

I. O. Barinov, E. S. Sedov, A. P. Alodjants and

S. M. Arakelian

Part C Nonlinear Optical Effects and Spectroscopy

FORMATION OF NARROW OPTICAL RESONANCES USING SUBMICRON-THIN ATOMIC VAPOR LAYERS

D. Sarkisyan and A. Papoyan

MODELLING MAGNETO-OPTICAL RESONANCES IN ATOMIC RUBIDIUM AT $D_{1}$ EXCITATION IN EXTREMELY THIN CELLS WHILE MAINTAINING A SELF-CONSISTENT SET OF THEORETICAL PARAMETERS

L. Kalvans, M. Auzinsh, R. Ferber, F. Gahbauer, A. Jarmola

A. Papoyan and D. Sarkisyan

LASER ISOTOPE SEPARATION IN ATOMIC VAPOUR. PHOTO-CHEMICAL METHOD VS. PHOTOIONIZATION ONE

P. A. Bokhan, N. V. Fateyev, D. E. Zakrevskiy, V. V. Buchanov and M. A. Kazaryan

TWO-DIMENSIONAL CONFINED TERAHERTZ WAVE PROPAGATION IN GAP PLASMON WAVEGUIDE FORMED BY TWO CYLINDRICAL SURFACES

Yu. H. Avetisyan, A. H. Manukyan, H. S. Hakobyan and T. N. Poghosyan 
xvi

BROADBAND SIMILARITON: FEATURES AND APPLICATIONS

A. Zeytunyan, A. Muradyan, G. Yesayan, L. Mouradian,

F. Louradour and A. Barthélémy

Index 